Draft Version NOVEMber 7, 2018

Preprint typeset using $\mathrm{LATE}_{\mathrm{E}} \mathrm{X}$ style AASTeX6 v. 1.0

\title{
UNRAVELING THE LINKS AMONG SYMPATHETIC ERUPTIONS
}

\author{
Dong Wang ${ }^{1,2}$, Rui Liu ${ }^{1}$, Yuming Wang ${ }^{1}$, Tingyu Gou ${ }^{1}$, Quanhao Zhang ${ }^{1}$, Zhenunn Zhou ${ }^{3}$, Min Zhang ${ }^{2}$ \\ ${ }^{1}$ CAS Key Laboratory of Geospace Environment, Department of Geophysics and Planetary Sciences, University of Science and Technology \\ of China, Hefei, Anhui 230026, China; rliu@ustc.edu.cn \\ ${ }^{2}$ Department of Mathematics and Physics, Anhui Jianzhu University, Hefei, Anhui 230601, China \\ ${ }^{3}$ School of Atmospheric Sciences, Sun Yat-sen University, Zhuhai, Guangdong 519000, China
}

\begin{abstract}
Solar eruptions occurring at different places within a relatively short time interval are considered to be sympathetic. However, it is difficult to determine whether there exists a cause and effect between them. Here we study a failed and a successful filament eruption following an X1.8-class flare on 2014 December 20, in which slipping-like magnetic reconnections serve as a key causal link among the eruptions. Reconnection signatures and effects are: at both sides of the filament experiencing the failed eruption, serpentine ribbons extend along chromospheric network to move away from the filament, while a hot loop apparently grows above it; at the filament undergoing the successful eruption, overlying cold loops contract, while coronal dimming appears at both sides even before the filament eruption. These effects are understood by reconnections continually transforming magnetic fluxes overlying one filament to the other, which adjusts how the magnetic field decays with increasing height above the filaments in opposite trends, therefore either strengthening or weakening the magnetic confinement of each filament.
\end{abstract}

Keywords: Sun: flares_-Sun: filaments_-Sun: coronal mass ejections - magnetic fields

\section{INTRODUCTION}

The most energetic phenomena in the solar atmosphere are flares, filament eruptions, and coronal mass ejections (CMEs). CME-caused disturbances and restructuring are often global (e.g., Hudson et al. 1996). Naturally the impact from a $\mathrm{CME}$ is expected to trigger the eruption of a nearby structure already on the verge of unstableness. But in practice it is hard to establish causal links between successive eruptions. This has been a key issue in research on sympathetic eruptions, eruptions that take place nearly synchronously in separated regions on the sun. Here the synchronicity is not meant in its literal sense but allows a short temporal separation of no more than a few hours (Moon et al. 2003).

The separated regions involved in sympathetic eruptions could be connected by large-scale coronal loops (e.g., Wang et al. 2001; Jiang et al. 2008) or by large-scale magnetic skeletons such as separators, separatrices, and quasiseparatrix layers (Schrijver \& Title 2011; Titov et al. 2012; Schrijver et al. 2013). The long-distance coupling may result from the impact of CMEs, waves, or propagating perturbations (e.g., Wang et al. 2001; Jiang et al. 2008; Török et al. 2011; Lynch \& Edmondson 2013; Jin et al. 2016). A crucial physical link between sympathetic eruptions is often proposed to be magnetic reconnection of large-scale magnetic field as induced indirectly or directly by distant or nearby eruptions: the reconnection may effectively modify the overlying field that provides the confining force for a pre-eruptive structure underneath, as suggested in numerous observational investigations (Liu et al. 2009a; Zuccarello et al. 2009; Jiang et al. 2011; Shen et al. 2012; Yang et al. 2012; Joshi et al. 2016; Wang et al. 2016) and corroborated by a few numerical simulations (Ding et al. 2006; Török et al. 2011; Lynch \& Edmondson 2013). The consequence of the reconnection is occasionally observed as coronal dimming (Jiang et al. 2011) when the overlying 
field is partially removed by reconnection. However, smoking-gun evidence for reconnections of large-scale magnetic field has seldom been reported in the literature, supposedly because such reconnections involve weaker magnetic field and are hence much less energetic than those in major flares and CMEs, therefore leaving only elusive signatures in the solar atmosphere.

In this paper, we present unambiguous evidence for magnetic reconnection of large-scale magnetic field involved in a set of sympathetic eruptions, including an X-class flare and its accompanying CME, a failed and a successful filament eruption. We conclude that the reconnection plays an important role in regulating the behavior of the filament eruptions. In the sections that follow, we analyze the observations in Section 2, and interpret the observations in Section 3.

\section{OBSERVATION \& ANALYSIS}

\subsection{Overview}

In this study we mainly used EUV images taken by the Atmospheric Imaging Assembly (AIA; Lemen et al. 2011) on-board the Solar Dynamics Observatory (SDO; Pesnell et al. 2012). AIA provides high resolution (1.5") and high cadence $(12 \mathrm{~s})$ full-disk images up to $1.28 \mathrm{R}_{\odot}$ at multiple passbands. AIA's EUV passbands cover a wide temperature range. Among them, 131, 94, and $335 \AA$ are usually sensitive to hot plasma in active regions; 171,193 , and $211 \AA$ respond well to "quiet" coronal loops; $304 \AA$ mainly covers the chromosphere and transition region. However, caution has to be taken because each passband contains multiple emission lines (Boerner et al. 2012).

The set of sympathetic eruptions under investigation include an X1.8-class flare from the NOAA active region (AR) 12242, a failed and a successful filament eruption originating from a quiet region located to the south of AR 12242 (Figure 1). The X1.8-class flare starts at 00:11 UT and peaks at 00:28 UT on 2014 December 20 (Figure 2a). In AIA $131 \AA$, an expanding bubble-like structure can be seen to erupt southwestward from AR 12242 (Figure 1c and accompanying animation). The eruptive structure make different effects on three nearby filaments, one is located at the south boundary of the active region (F1 hereafter) and the other two are quiescent filaments (F2 and F3 hereafter) located to the further south (Figure $1(\mathrm{a} \& \mathrm{~b})$ ). F1 is close to the flare site, but remains intact during the flare. F2 is apparently disturbed by the eruptive bubble, but it fails to erupt. F3 initially survives the X-class flare, but becomes unstable later and erupts during the decay phase of the flare.

To establish the timeline, we constructed a time-distance map (Figure 2b) to show the ejection associated with the X-class flare seen through a virtual slit S1 (Figure 1c), and a similar map (Figure 2c) to show the successful filament eruption seen through a virtual slit S2 (Figure 1d). With the soft X-ray 1-8 A flux given by Geostationary Operational Environmental Satellite (GOES; Figure 2a), one can see that the earlier eruption is synchronized with the X-class flare, but the later one is delayed by half an hour. Employing the Large Angle Spectroscopic Coronagraph (LASCO) on-board the Solar and Heliospheric Observatory (SOHO), we identified both the earlier CME associated with the X-class flare (Figure 1e) and the later one resulting from the successful filament eruption (Figure 1f, see also Figure 2a). As the later CME closely trails the earlier one, they are registered as one single event in the SOHO LASCO CME catalog. The two CMEs occur well within the temporo-spatial range that is determined by the Alfvén speed, typically on the order of $1000 \mathrm{~km} \mathrm{~s}^{-1}$ in the corona, since the time difference between the onset of the X-class flare and F3's eruption is about $40 \mathrm{~min}$, and the distance from the flare to F3 is about 400 Mm, as measured along the spherical surface (Figure 1a). Thus a propagation speed over $170 \mathrm{~km} \mathrm{~s}^{-1}$ is required for the agent coupling the two events. Since this speed is much smaller than the typical Alfvén speed or the earlier CME's speed $\left(\sim 800 \mathrm{~km} \mathrm{~s}{ }^{-1}\right.$; Figure 2a), it is unlikely that the later CME is directly caused by the earlier one.

\subsection{Magnetic Configuration}

To understand the large-scale magnetic configuration, we utilized the pfss package in SolarSoftWare, which reconstructs the coronal field with a potential-field source-surface model (PFSS; Schatten et al. 1969). To approximate the evolving field on the full sphere, the lower boundary of the PFSS model, i.e., the synoptic map of the photospheric magnetic field, is updated every 6 hours by assimilating magnetograms into a flux-dispersal model (Schrijver \& DeRosa 
2003).

With a pre-flare PFSS model of the coronal magnetic field (Figure 3), we calculated the decay index, $n=$ $-d \ln B / d \ln h$, right above the three filaments by manually picking points along each filament visible in $304 \AA$ (Figure $1(\mathrm{a} \& \mathrm{~b}))$. The decay index describes how fast the magnetic field decreases with increasing height. Kliem \& Török (2006) found that a toroidal flux ring is unstable to lateral expansion if the decay index of the external poloidal field $B_{\text {ex }}$ exceeds $3 / 2$ (hence the term torus instability). Usually one cannot decouple $B_{\text {ex }}$ from the flux-rope field, but can approximate $B_{\text {ex }}$ with a potential field (e.g., Török \& Kliem 2007; Démoulin \& Aulanier 2010; Wang et al. 2017). This is because the external field component orthogonal to the flux-rope axis effects the downward $\mathbf{J} \times \mathbf{B}$ force, and potential field is almost orthogonal to the polarity inversion line (PIL), with which the flux rope in equilibrium is typically aligned. Hence in our calculation $n=-d \ln B_{h} / d \ln h$, where $B_{h}$ is the horizontal component perpendicular to the radial component $B_{r}$ in spherical coordinates.

Figure 4 shows the variation of decay index as a function of height averaged for the selected points of each filament, and the critical height $h_{\text {crit }}$ (Wang et al. 2017) corresponding to the theoretical threshold $\left(n_{\text {crit }}=1.5\right)$ of the torus instability. One can see that all three filaments are quite stable to the torus instability, with $h_{\text {crit }}$ exceeding $200 \mathrm{Mm}$ $\left(\sim 0.3 R_{\odot}\right)$ for the two quiescent filaments F2 and F3 and exceeding $100 \mathrm{Mm}$ for the AR filament F1. As a comparison, using triangulation methods, Xu et al. (2010) and Liu et al. (2012b) found a few AR filaments lying below $35 \mathrm{Mm}$ $\left(0.05 R_{\odot}\right)$ before eruptions; Liu et al. (2013) found a few quiescent filaments lying below $70 \mathrm{Mm}$. These numbers agree with a statistics showing that eruptive prominences typically become unstable in a height range 0.06-0.14 $R_{\odot}$ (Liu et al. 2012a).

In particular $\mathrm{F} 1$ has a saddle-like $n(h)$ profile, which may provide additional confinement at altitudes corresponding to small decay indexes at the bottom of the saddle (Wang et al. 2017). We also calculated F1's decay index in a potential field based only on $B_{z}$ of the active region, provided by hmi.sharp_cea data series from Helioseismic and Magnetic Imager (HMI; Schou et al. 2011; Scherrer et al. 2012) on-board SDO, but found that $n(h)$ increases monotonously with height (red curve in the left panel of Figure 4); this approach gives $h_{\text {crit }}=61 \mathrm{Mm}$, still quite large for an AR filament. The difference between the two $n(h)$ profiles for F1 demonstrates that the high-altitude magnetic field is strongly modulated by the quiet-Sun field outside the active region. The preflare background field may account for F1 and F2's behavior in response to the X-class flare, but cannot explain F3's successful eruption which is significantly delayed relative to the flare. Below we investigate the physical processes linking the flare and the filament eruptions.

\subsection{Slipping-Like Magnetic Reconnection}

An outstanding feature that precedes F3's eruption is a serpentine brightening on the surface propagating from F2 towards F3 in AIA $304 \AA$ (Figure 5 and accompanying animation), which apparently triggers the successful filament eruption when it approaches F3. Comparing AIA 304 and $1600 \AA$ images, one can see that the serpentine ribbon slips along the chromospheric network, where the magnetic field (plage field hereafter) is generally stronger than the intranetwork field. This indicates that magnetic field plays a key role here, unlike the surface brightening caused by filament material draining under gravity (e.g., Gilbert et al. 2013). A similar serpentine ribbon slips northward from F2 toward F1, when F2 becomes fractured in the middle with material moving towards both ends (Figure 6 and accompanying animation). F2 disappears several hours later due presumably to the material drainage to the surface. Meanwhile, a weak brightening point on the southern side of F3 appears to extend northward into a ribbon-like feature (bottom panels of Figure 6).

In tandem to the extension of the serpentine ribbon toward F3, a loop overlying F2 appears at about 00:37 UT in $131 \AA$, apparently rising and expanding, as demonstrated by the snapshots in Figure $7(\mathrm{a}-\mathrm{c})$ and accompanying animation, as well as the stack plot (Figure 2d) generated by a virtual slit S3 across the loop (Figure 7b). The loop's southern leg is less visible than its northern counterpart, and most of the time both footpoints cannot be clearly discerned, only at 00:40:20 UT can its southern footpoint be identified as a brightening feature to the north of F3 (marked by a red arrow in Figure 7a). This loop is diffuse and only visible in $131 \AA$, suggesting that it is heated to as hot as $\sim 10 \mathrm{MK}$. It continues to be visible and rising until F3's eruption.

In contrast to the hot loop, a bundle of large-scale cold loops in $171 \AA$ overlying both F2 and F3 are observed to 
contract toward F3 when F3 begins to erupt (Figure 7(d-f) and accompanying animation). In view of the PFSS model (Figure 3), this loop bundle corresponds to the large-scale arcade that overarches both the active region and the two quiescent filaments. It is anchored in the negative-polarity plage to the southeast of AR 12242 and the positive-polarity plage to the west of AR 12242 (see also Figure 1a). The stack plot generated by the virtual slit S4 across these loops gives a contraction speed of $30 \sim 40 \mathrm{~km} \mathrm{~s}^{-1}$ (Figure 2e).

The cold loop contraction and hot loop expansion during about 00:40-01:00 UT are also associated with coronal dimming which extends southward from the active region toward F3 (Figure 8). Visible in base-difference images of all three AIA passbands sensitive to the quiet corona, i.e., 171, 193, and $211 \AA$, the dimming indicates mass evacuation. Starting at about the flare peak time (Figure 2), the dimming may initially be associated with the eruption from the active region, but later it extends over F3 to its southern side (Figure 8 and accompanying animation). Before F3's eruption, the rapid decrease in brightness (Figure 2f) at two representative dimming points on both sides of F3 (marked by a red and green cross in Figure 8) is temporally associated with the ongoing expansion of hot loops (Figure 2d) and contraction of cold loops (Figure 2e).

\section{DISCUSSION \& CONCLUSION}

To account for the observational features presented above, a schematic diagram is given in Figure 9, based on the well-known fact that filaments are aligned along polarity inversion lines. For simplicity we have omitted AR 12242 and F1 but focus on F2 and F3, conjecturing that F1 lies low under strong magnetic confinement (see §2.2) so that it is not affected by the CME-associated disturbances from the active region (e.g., Liu et al. 2013). We have also neglected four complications. First, the positive-polarity field between F2 and F3 belongs to a large tract of plage field that extends to far west (Figure 1a). This plage field, together with the negative-polarity plage field to the southeast of AR 12242, dominates the large-scale magnetic connections in the southwest quadrant of the Sun, as demonstrated by the PFSS model (Figure 3) and the large-scale loops in AIA $171 \AA$ (Figure $7(\mathrm{~d}-\mathrm{f})$ ). Second, the field to the south of F3 is very weak and may possess severely mixed polarities, because the south polar field reversed signs i.e., became negative, only nine months before (Sun et al. 2015). Third, F2 and F3 are not parallel and F3 is curved. Fourth, white-light coronagraph images show a large-scale structure fanning out over the southwest quadrant with numerous plasma sheet extensions and persisting for many days (not shown), indicating ample presence of open field. But in the PFSS model, only a few open field lines (white) originate from the far west (Figure 3). Thus, the actual magnetic configuration must be much more complicated than that depicted in the cartoon.

With the above simplifications, the initial magnetic configuration is locally tripolar (Figure 9a), reminiscent of a pseudo streamer that overlies two loop arcades side by side, with each occasionally harboring a filament (Wang et al. 2007; Török et al. 2011). Magnetic reconnection in such a topology often involve the quasi-separatrix layer separating the two arcades (e.g., Liu et al. 2014; Gou et al. 2016). Similarly in our case, one can see that PFSS field lines traced from the positive-polarity plage field between F2 and F3 are mapped to distant places, overlying F2 and F3 separately (Figure 9d), which indicates the presence of quasi-separatrix layers (Démoulin 2006). Here magnetic reconnection is speculated to be triggered by the CME associated with the X-class flare and to occur between field lines overlying F3 and some higher field lines. The reconnection produces new field lines (red) overlying F2 and those anchored on the right (southern) side of F3, the latter of which are either open, if the higher field lines are open (e.g., Török et al. 2011); or connected to remote places, most likely to the positive-polarity plage in the far west, if the higher field lines are closed. Similar reconnections take place successively (Figure 9(b \& c)), manifesting themselves as the slipping extension of serpentine ribbons along the chromospheric network away from both sides of F2 (Figures 5 and 6) and

the apparent rising and expansion of hot loops overlying F2 in $131 \AA$ (Figures $2 \mathrm{~d}$ and $7(\mathrm{a}-\mathrm{c})$ ). A similar slippage of surface brightening toward F3 is expected on its right (southern) side (indicated by a dashed arrow in Figure 9c), but only weak signatures are seen in $304 \AA$ (bottom panels of Figure 6 ). This is partly attributed to the reduced imaging contrast closer to the limb, with structures being 'squeezed' into a narrower space by projection. On the other hand, the surface brightening is expected to be weak if open field lines result from the reconnections. These reconnections are termed "slipping-like", because it is impossible to determine whether the field lines slip through plasma at sub- or super-Alfvénic speeds, which is termed slipping or slip-running reconnection (Aulanier et al. 2006, 2007).

As the slipping-like reconnections effectively open F3's overlying field, the coronal plasma above F3 would be evacuated, which predicts coronal dimming at both sides of F3 (Figure 9c). This is indeed observed in AIA 171, 193, 
and $211 \AA$ before F3's eruption (Figures $2 \mathrm{f}$ and 8). Meanwhile, large-scale overlying loops would sense a temporary reduction in magnetic pressure above F3 due to the flux and mass evacuation, and hence contract toward F3 to seek a new equilibrium, as observed in AIA $171 \AA$ (Figures 2e and $7(\mathrm{~d}-\mathrm{f})$ ). This so-called "coronal implosion" works in a wide range of flare phenomena (e.g., Liu \& Wang 2009, 2010; Liu et al. 2009b, 2012c; Gou et al. 2017). Most importantly, the slipping-like reconnections continually strengthen the magnetic force confining F2 while weakening the force confining F3 until F3 becomes unstable to the torus instability and erupts.

The mechanism proposed here is not new, but to our knowledge signatures and effects of magnetic reconnection have seldom been identified in the literature on sympathetic eruptions, hence our results substantiate magnetic reconnection of large-scale field as a key causal link among sympathetic eruptions. These observations also highlight the ubiquity of magnetic reconnection by demonstrating its efficacy in the large-scale field of the quiet-Sun corona, where the magnetic field is weak and supposed to be approximately free of current, yet magnetic reconnection releases enough magnetic energy to heat up the chromosphere and newly reconnected coronal loops.

D.W. acknowledges support by Natural Science Foundation of Anhui Province Education Department (KJ2017A493, KJ2017A491, gxyq2018030) and NSFC 11704003. R.L. acknowledges support by NSFC 41474151, 41774150, and 41761134088. Y.W. acknowledges support by NSFC 41774178 and 41574165 . M.Z. acknowledges support by Natural Science Foundation of Anhui Province Education Department (KJ2016JD24) and Anhui Province Quality Engineering (2017zhkt161). This work is also supported by NSFC 41421063, CAS Key Research Program of Frontier Sciences QYZDB-SSW-DQC015, and the fundamental research funds for the central universities.

\section{Software: SolarSoftWare (Freeland \& Handy 2012)}

\section{REFERENCES}

Aulanier, G., Pariat, E., Démoulin, P., \& DeVore, C. R. 2006, SoPh, 238, 347

Aulanier, G., Golub, L., DeLuca, E. E., et al. 2007, Science, 318, 1588

Boerner, P., Edwards, C., Lemen, J., et al. 2012, SoPh, 275, 41

Démoulin, P. 2006, Advances in Space Research, 37, 1269

Démoulin, P., \& Aulanier, G. 2010, ApJ, 718, 1388

Ding, J. Y., Hu, Y. Q., \& Wang, J. X. 2006, SoPh, 235, 223

Freeland, S. L., \& Handy, B. N. 2012, SolarSoft: Programming and data analysis environment for solar physics, Astrophysics Source Code Library, , , ascl:1208.013

Gilbert, H. R., Inglis, A. R., Mays, M. L., et al. 2013, ApJL, 776, L12

Gou, T., Liu, R., Wang, Y., et al. 2016, The Astrophysical Journal Letters, 821, L28

Gou, T., Veronig, A. M., Dickson, E. C., Hernandez-Perez, A., \& Liu, R. 2017, ApJL, 845, L1

Hudson, H. S., Acton, L. W., \& Freeland, S. L. 1996, ApJ, 470, 629

Jiang, Y., Shen, Y., Yi, B., Yang, J., \& Wang, J. 2008, ApJ, 677, 699

Jiang, Y., Yang, J., Hong, J., Bi, Y., \& Zheng, R. 2011, ApJ, 738,179

Jin, M., Schrijver, C. J., Cheung, M. C. M., et al. 2016, ApJ, 820,16

Joshi, N. C., Schmieder, B., Magara, T., Guo, Y., \& Aulanier, G. 2016, ApJ, 820, 126

Kliem, B., \& Török, T. 2006, Physical Review Letters, 96, 255002

Lemen, J. R., Akin, D. J., Boerner, P. F., et al. 2011, in The Solar Dynamics Observatory (Springer), 17-40

Liu, C., Lee, J., Karlický, M., et al. 2009a, ApJ, 703, 757

Liu, K., Wang, Y., Shen, C., \& Wang, S. 2012a, ApJ, 744, 168

Liu, R., Kliem, B., Török, T., et al. 2012b, ApJ, 756, 59
Liu, R., Liu, C., Török, T., Wang, Y., \& Wang, H. 2012c, ApJ, 757,150

Liu, R., Liu, C., Xu, Y., et al. 2013, ApJ, 773, 166

Liu, R., Titov, V. S., Gou, T., et al. 2014, ApJ, 790, 8

Liu, R., \& Wang, H. 2009, ApJL, 703, L23

-. 2010, ApJL, 714, L41

Liu, R., Wang, H., \& Alexander, D. 2009b, ApJ, 696, 121

Lynch, B. J., \& Edmondson, J. K. 2013, ApJ, 764, 87

Moon, Y.-J., Choe, G. S., Wang, H., \& Park, Y. D. 2003, ApJ, 588,1176

Pesnell, W. D., Thompson, B. J., \& Chamberlin, P. C. 2012, SoPh, 275, 3

Schatten, K. H., Wilcox, J. M., \& Ness, N. F. 1969, SoPh, 6, 442

Scherrer, P. H., Schou, J., Bush, R., et al. 2012, Solar Physics, 275, 207

Schou, J., Scherrer, P., Bush, R., et al. 2011, in The Solar Dynamics Observatory (Springer), 229-259

Schrijver, C. J., \& DeRosa, M. L. 2003, Solar Physics, 212, 165

Schrijver, C. J., \& Title, A. M. 2011, Journal of Geophysical Research (Space Physics), 116, A04108

Schrijver, C. J., Title, A. M., Yeates, A. R., \& DeRosa, M. L. 2013, ApJ, 773, 93

Shen, Y., Liu, Y., \& Su, J. 2012, ApJ, 750, 12

Sun, X., Hoeksema, J. T., Liu, Y., \& Zhao, J. 2015, ApJ, 798, 114

Titov, V. S., Mikic, Z., Török, T., Linker, J. A., \& Panasenco, O. 2012, ApJ, 759, 70

Török, T., \& Kliem, B. 2007, Astronomische Nachrichten, 328, 743

Török, T., Panasenco, O., Titov, V. S., et al. 2011, ApJL, 739, L63

Wang, D., Liu, R., Wang, Y., et al. 2017, The Astrophysical Journal Letters, 843, L9

Wang, H., Chae, J., Yurchyshyn, V., et al. 2001, ApJ, 559, 1171 
Wang, R., Liu, Y. D., Zimovets, I., et al. 2016, ApJL, 827, L12

Wang, Y.-M., Sheeley, Jr., N. R., \& Rich, N. B. 2007, ApJ, 658, 1340

Xu, Y., Jing, J., \& Wang, H. 2010, SoPh, 264, 81
Yang, J., Jiang, Y., Zheng, R., et al. 2012, ApJ, 745, 9

Zuccarello, F., Romano, P., Farnik, F., et al. 2009, A\&A, 493, 629 

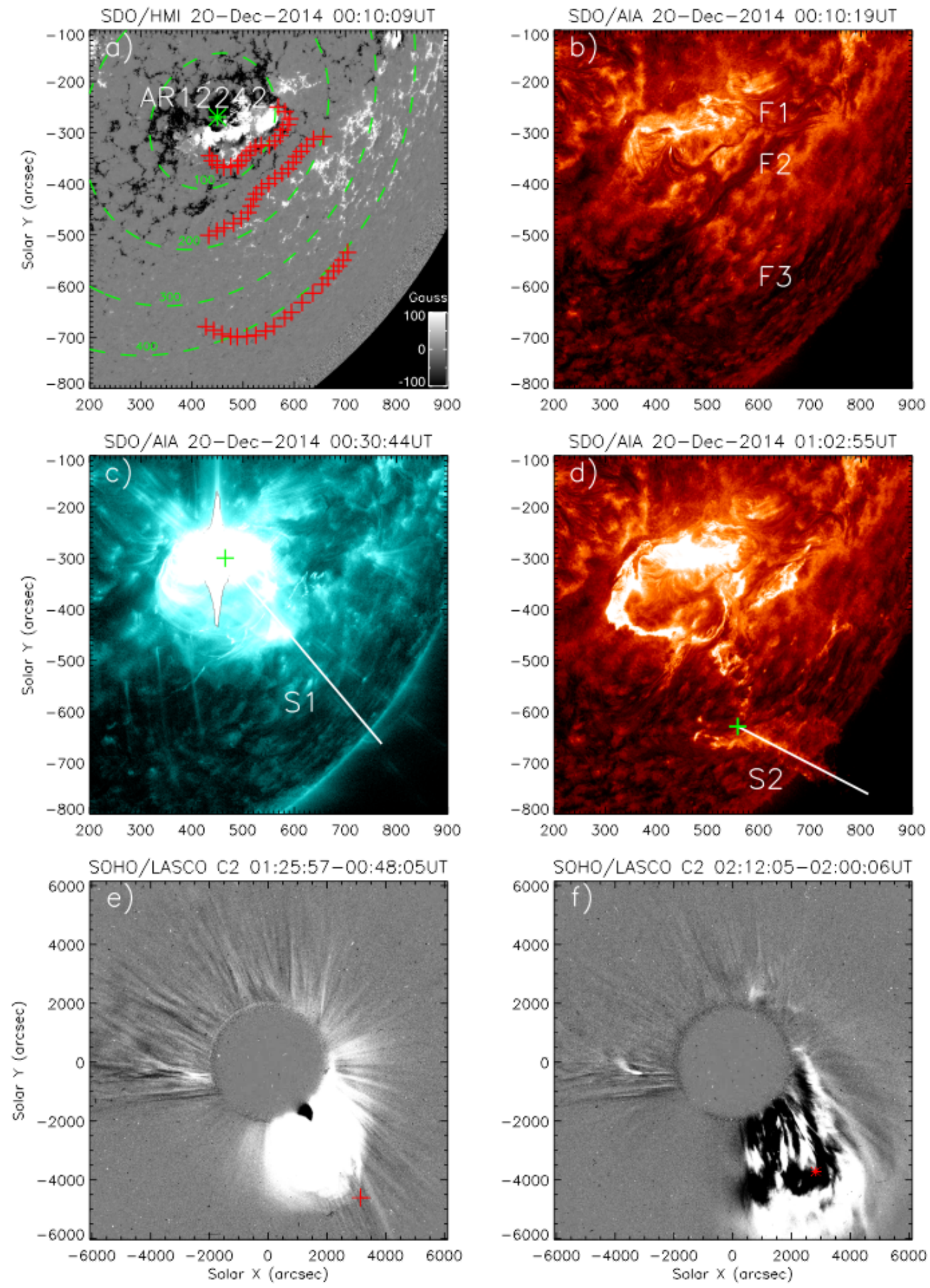

Figure 1. Overview of the sympathetic eruptions. a) shows the southwest quadrant of a line-of-sight HMI magnetogram before the X1.8-class flare. Red plus symbols mark the locations of the three filaments, F1, F2, and F3, in the corresponding AIA $304 \AA$ image in b). Green dashed circles indicate the distance measured along the surface of the sphere from the center of the active region (marked by a green asterisk) in units of Mm. The flare is shown in AIA $131 \AA$ in c), and F3's eruption in AIA $304 \AA$ in d). Their corresponding CMEs are observed by LASCO C2 in e) and f), respectively, with the former CME front marked by a red plus, and the later by a red asterisk. Two virtual slits, S1 and S2, are indicated in (c) and (d), respectively, and their starting points are marked by green plus symbols. An animation of AIA 131 and $304 \AA$ Amages is available online. 


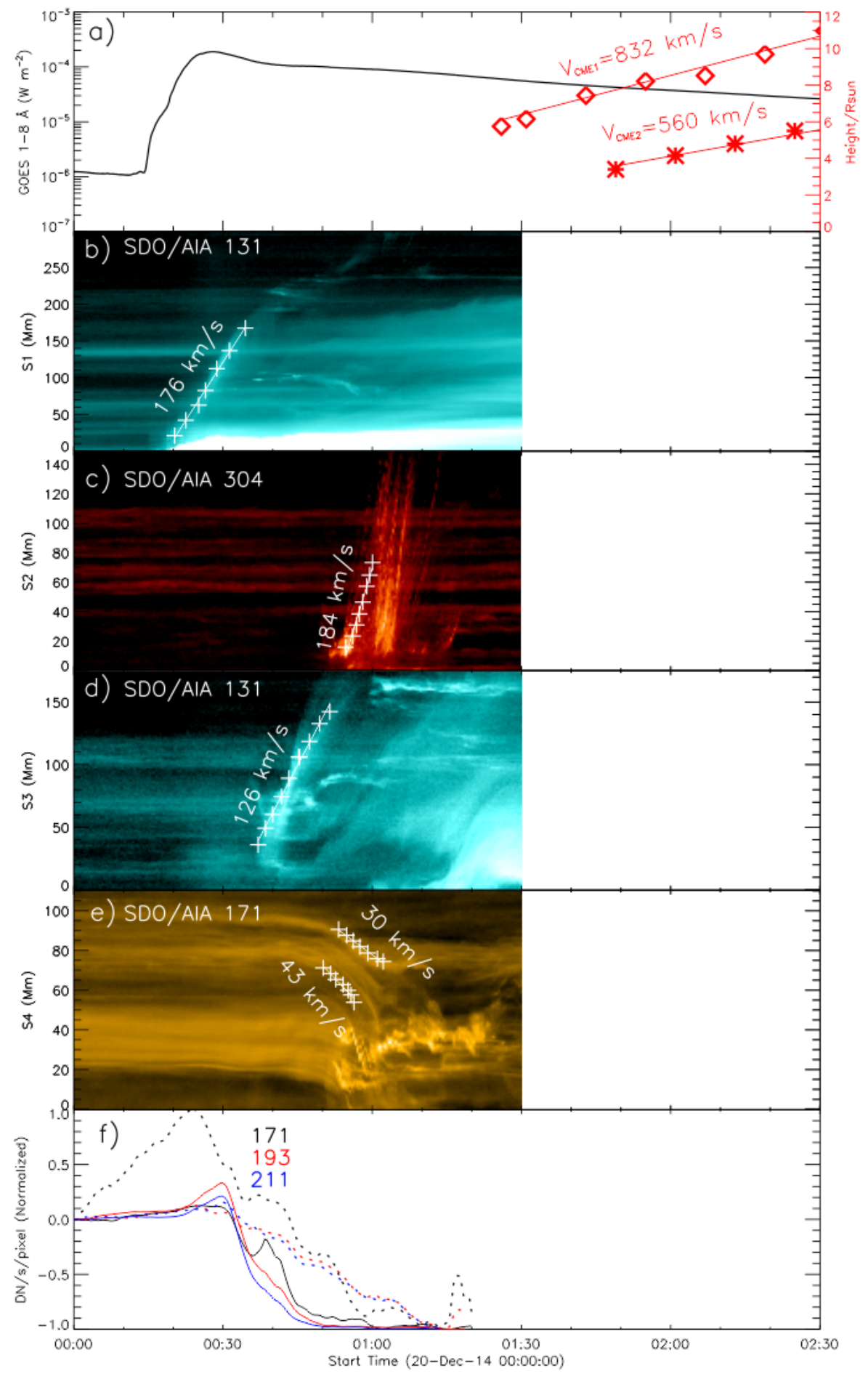

Figure 2. Timeline of the sympathetic eruptions. Panel a) shows GOES 1-8 $\AA$ light curve, scaled by the left $y$-axis; and heights of the two successive CMEs as a function of time, measured with LASCO's C2 and C3 cameras and scaled by the right $y$-axis. Panels (b-e) show stack plots constructed with the virtual slits S1-S4 as indicated in Figures 1c, 1d, 7b, and 7d, respectively. Linear fittings of the features marked by white crosses in the stack plots give the projected speeds of the eruptive structure associated with the X1.8-class flare (b), the filament eruption (c), the apparent rising and expansion of the hot loop (d), and the contraction of the cold loops (e). Panel (f) shows the variation of normalized mean brightness in two $20^{\prime \prime} \times 20^{\prime \prime}$ representative dimming regions marked in the bottom-middle panel of Figure 8. The solid (dotted) lines correspond to the region on the northern (southern) side of F3. 

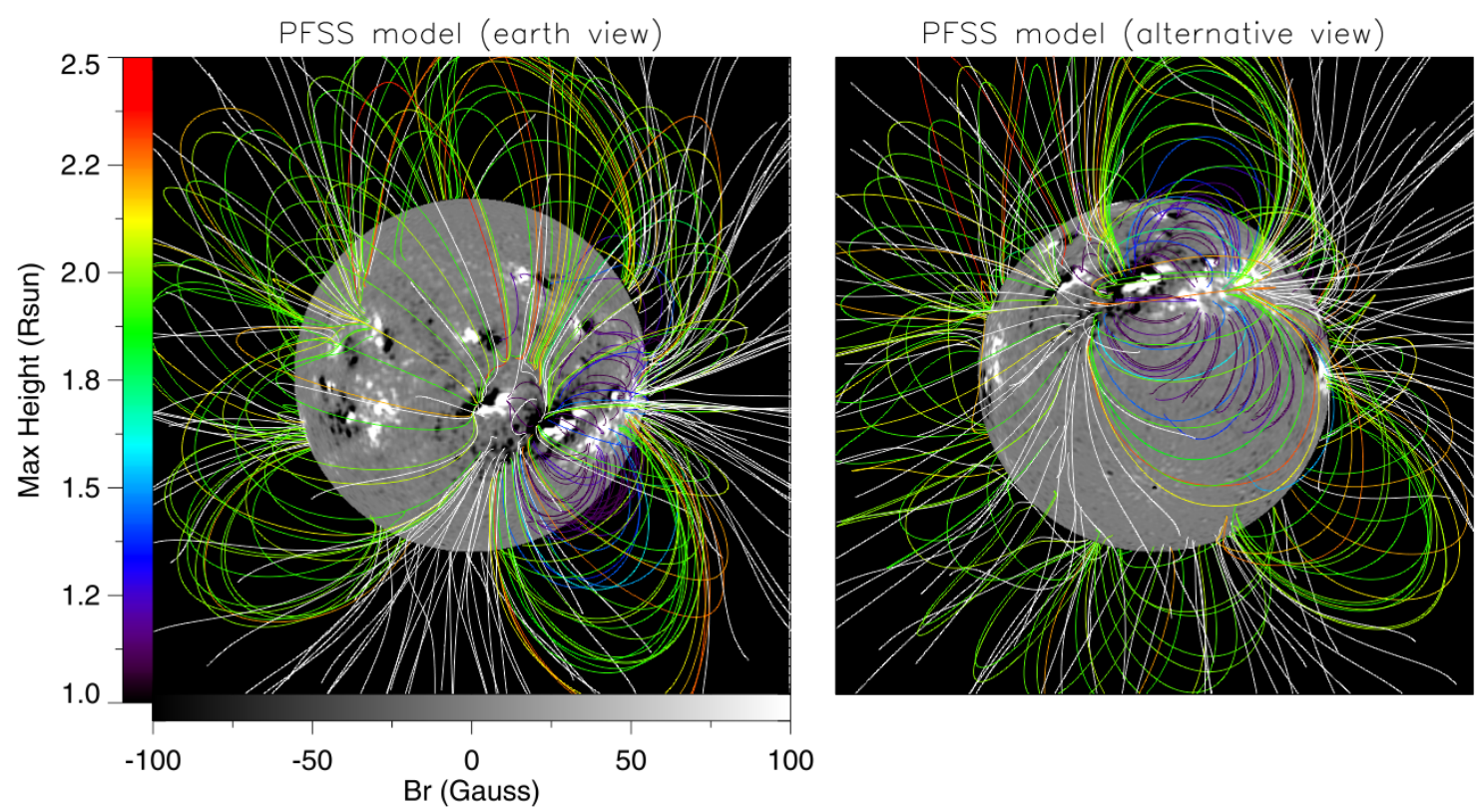

Figure 3. Coronal magnetic field reconstructed with the PFSS model. The plots combine the large-scale field lines around the globe and small-scale field lines in the southwest quadrant where AR 12242 is located. Open field lines are shown in white. Closed field lines are colored by each own maximum height, and viewed from the Earth (left) and from a southern perspective (right).
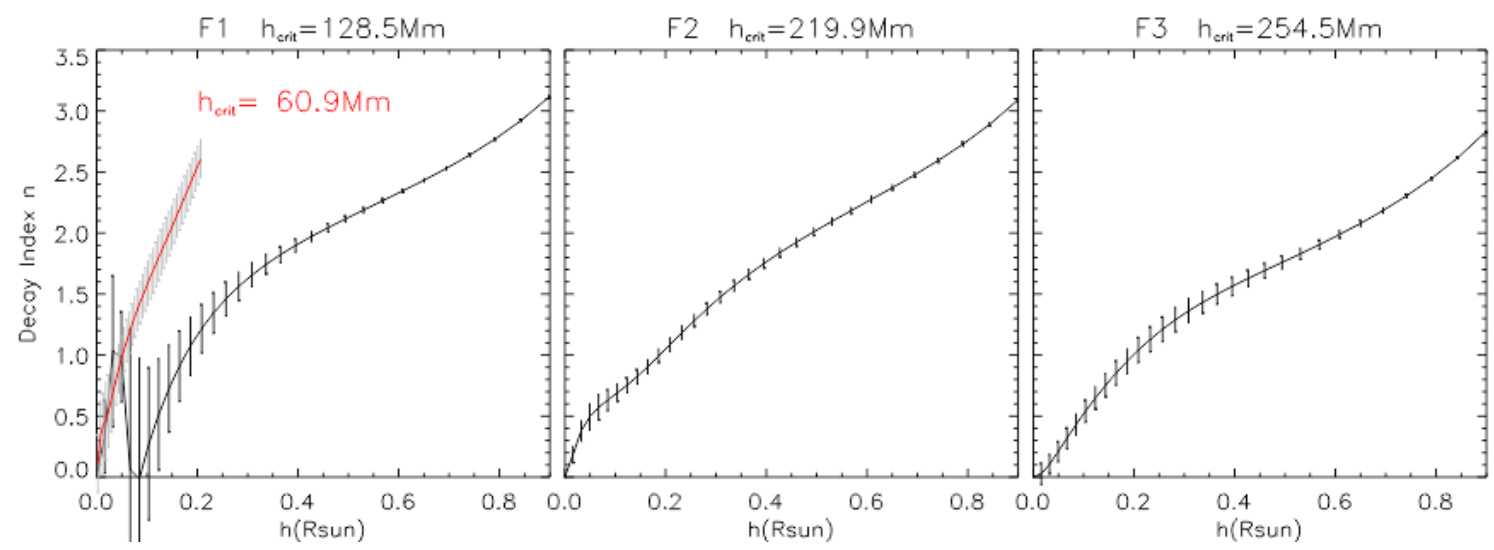

Figure 4. Decay index as a function of height above the three filaments of interest. The height is given in units of $R_{\text {sun }}$ above the surface. For F1, the decay index is calculated with both a PFSS model (black) and a local potential extrapolation (red). The error bars result from standard deviation of the manually picked points shown in Figure 1. 


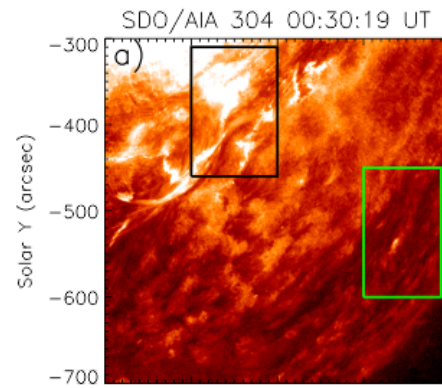

SDO/AIA 304 00:31:55 UT

SDO/AIA 304 00:33:31 UT

SDO/AIA 304 00:49:19 UT
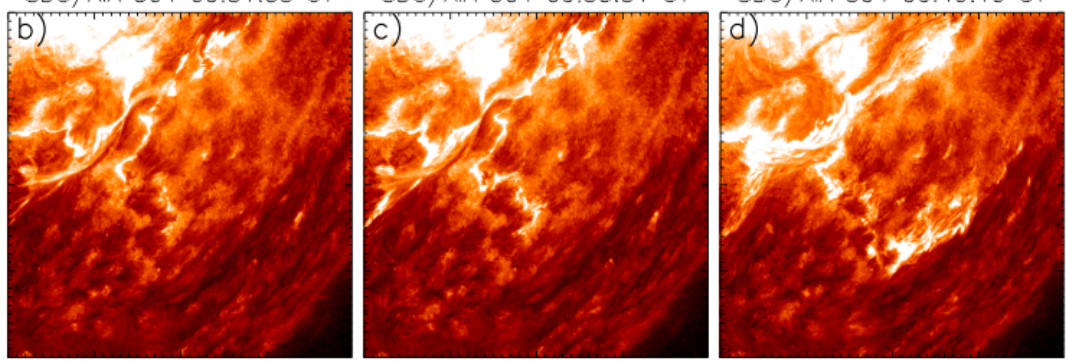

SDO/AIA 1600 00:30:16 UT SDO/AIA 1600 00:31:52 UT
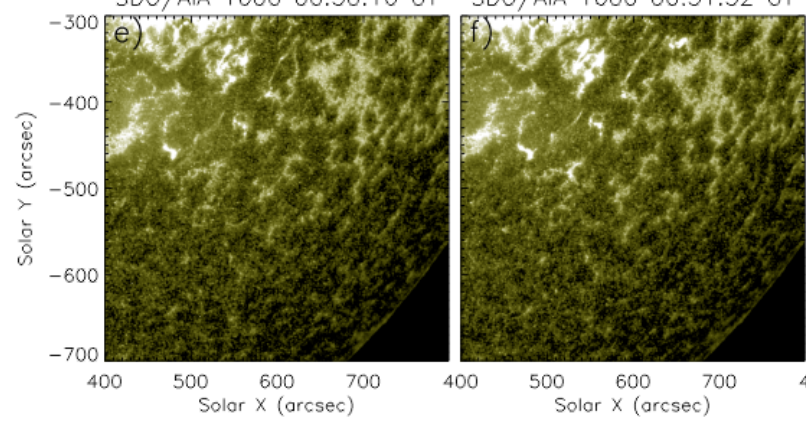

SDO/AIA 1600 00:33:28 UT

SDO/AIA 1600 00:49:52 UT
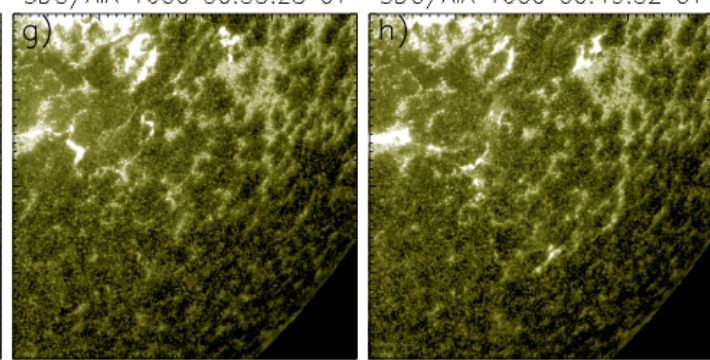

$500 \quad 600700$
Solor $\times(\operatorname{arcsec})$

$400 \quad 500 \quad 600 \quad 700$

$400 \quad 500 \quad 600 \quad 700$

Figure 5. Slipping extension of serpentine ribbons at both sides of F2. A black box marks the brightening to the north of F2, a green box marks a weak brightening to the south of F3. Figure 6 zooms in on both regions. An animation of AIA 1600 and $304 \AA$ images is available online, with corresponding base-difference images. 


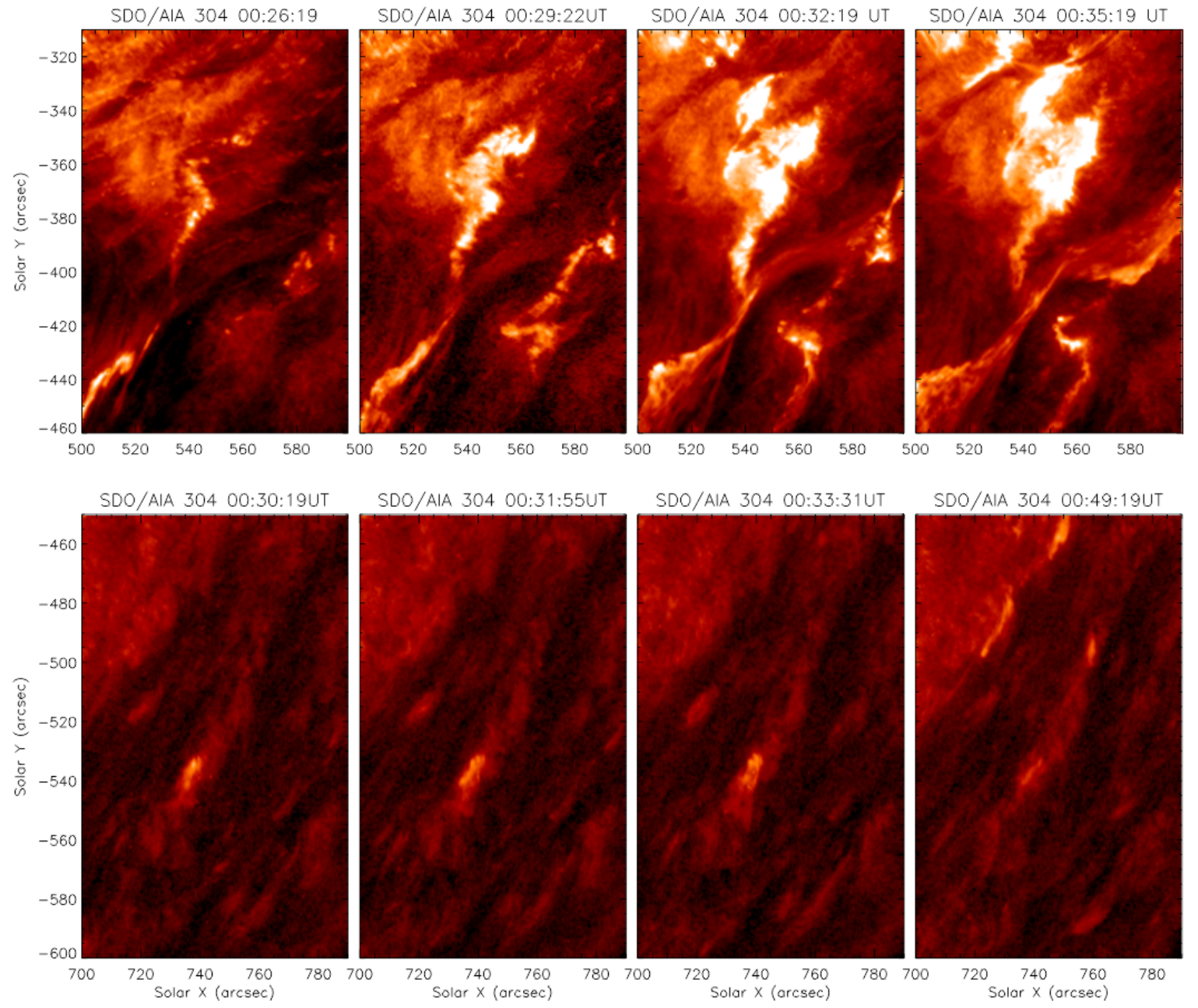

Figure 6. Slipping brightening at far sides of F2 and F3. Top panels show the slipping extension of a serpentine ribbon to the northward of F2. Bottom panels show that a weak brightening to the south of F3 extends northward. The top (bottom) panels' field of view is indicated by the black (green) rectangle in Figure 5a. An animation of AIA $304 \AA$ images with a larger FOV is available online to show F2's failed eruption. 

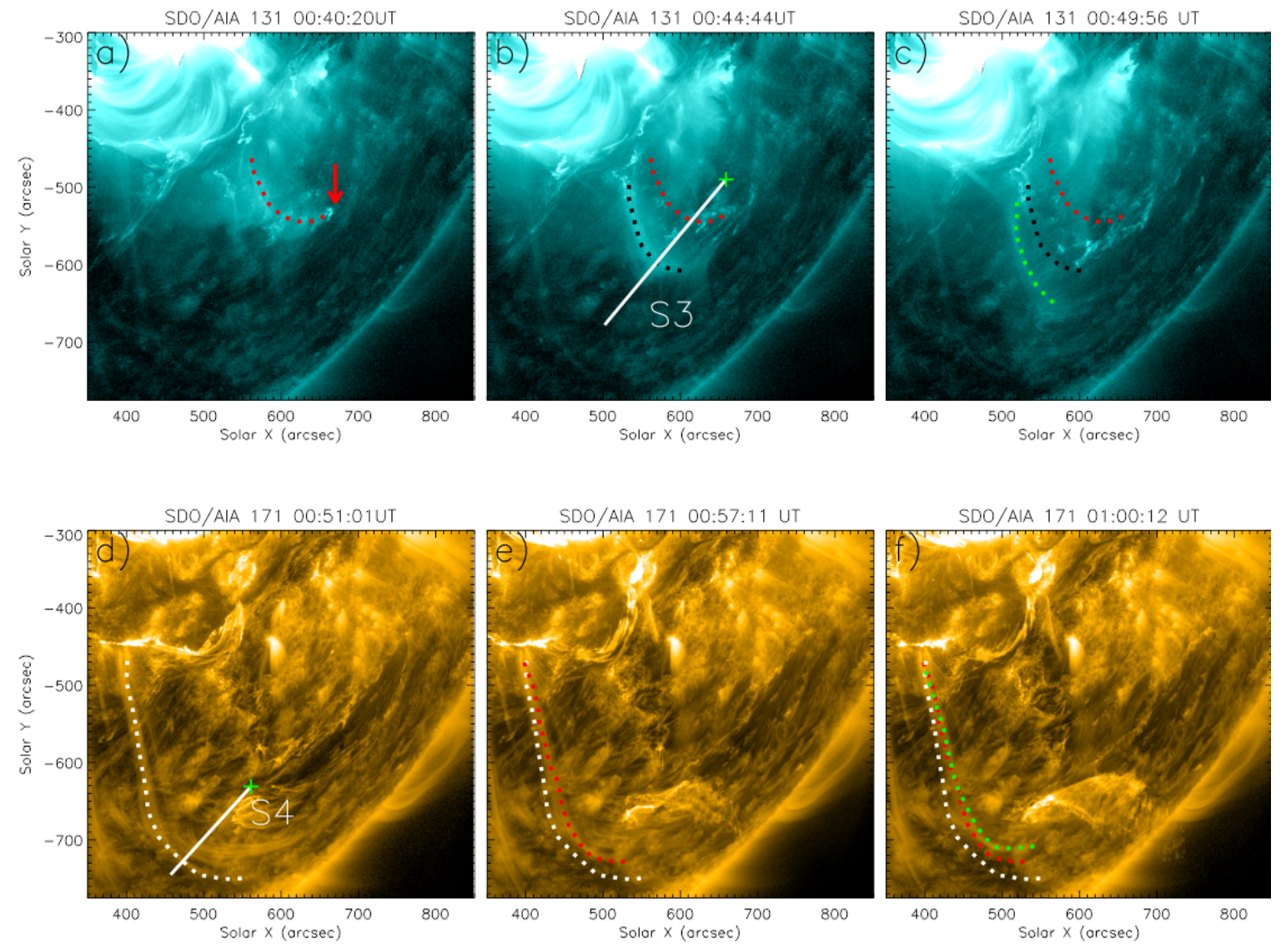

Figure 7. Loop dynamics associated with the slipping-like reconnection. (a-c) Loop rising and expansion observed in AIA $131 \AA$. The red, black and green dotted curves delineate the loop shape at 00:40, 00:44, and 00:49 UT, respectively. The red arrow in (a) marks the footpoint brightening of the loop. (d-f) Loop contraction observed in AIA $171 \AA$. The white, red and green dotted curves delineate the loop shape at 00:51, 00:57, and 01:00 UT, respectively. Two virtual slits, S3 and S4, are indicated in (b) and (d), respectively, and their starting points are marked by green plus symbols. An animation of A1A 131 and $171 \AA$ images is available online. 

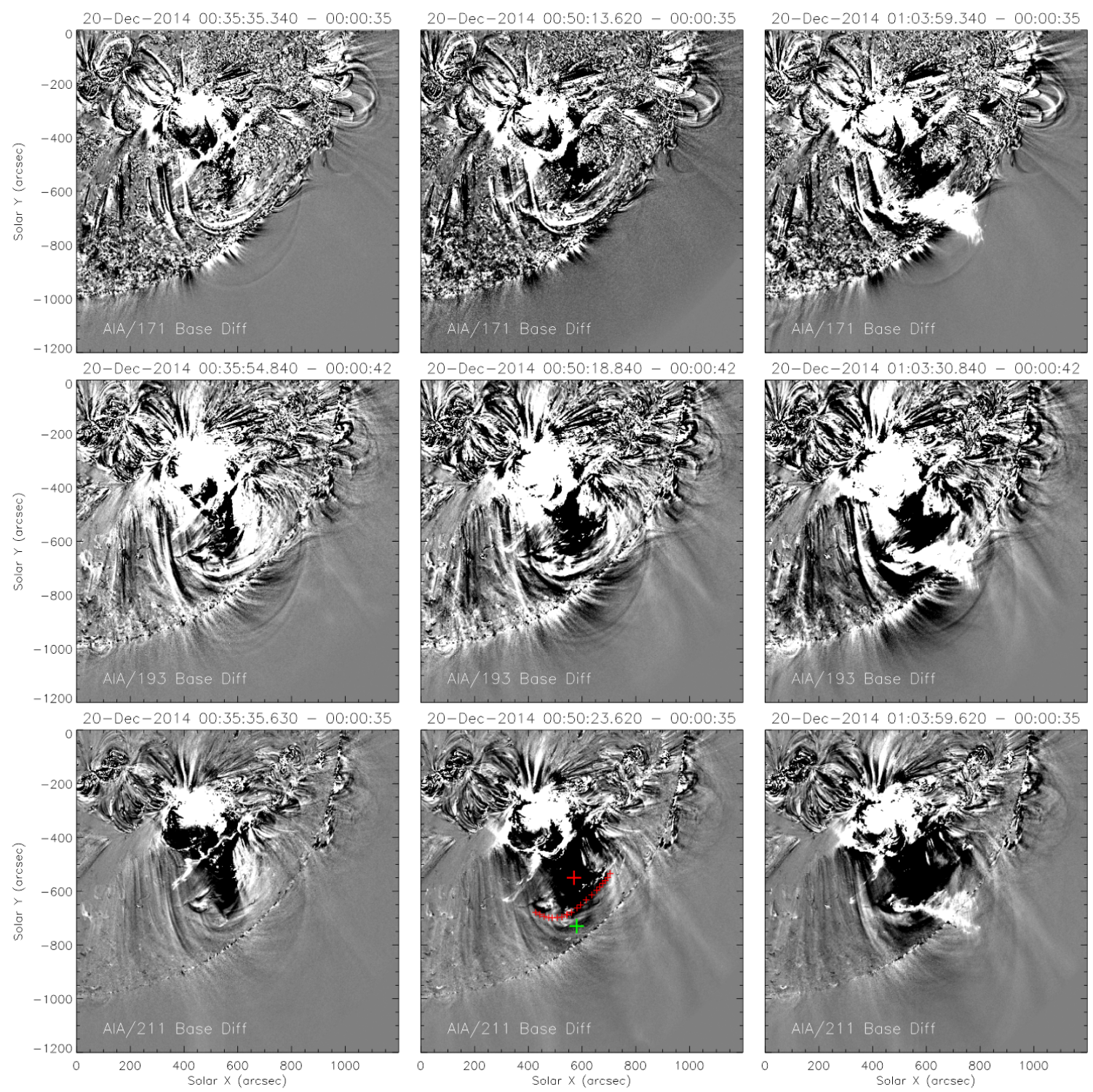

Figure 8. Coronal dimming in the sympathetic eruptions. The snapshots show base-difference images in $171 \AA$, $193 \AA$, and $211 \AA$. An online animation is available showing the original, base- and running-difference images in the three AIA passbands. In the bottom-middle panel, the location of F3 is marked by ' + ' symbols, same as in Figure la; the red (green) marks on the northern (southern) side of $\mathrm{F} 3$ the center of a $20^{\prime \prime} \times 20^{\prime \prime}$ region, from which the light curves in Figure $2 \mathrm{f}$ are derived. 
a)

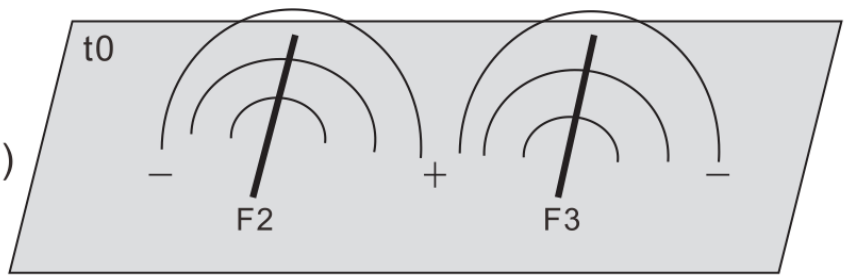

b)

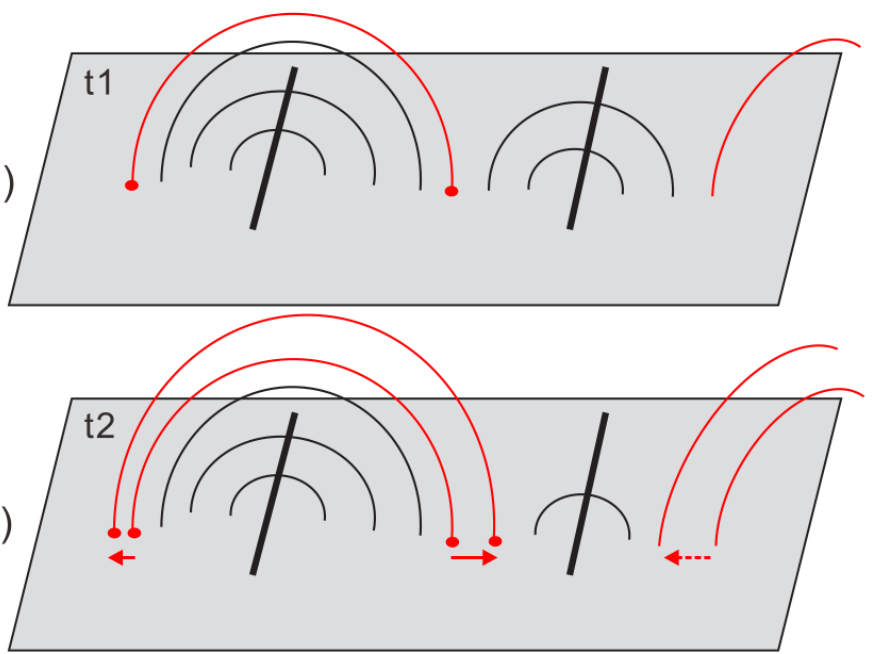

d) SDO/HMI 20-Dec-2014 00:10:09 UT

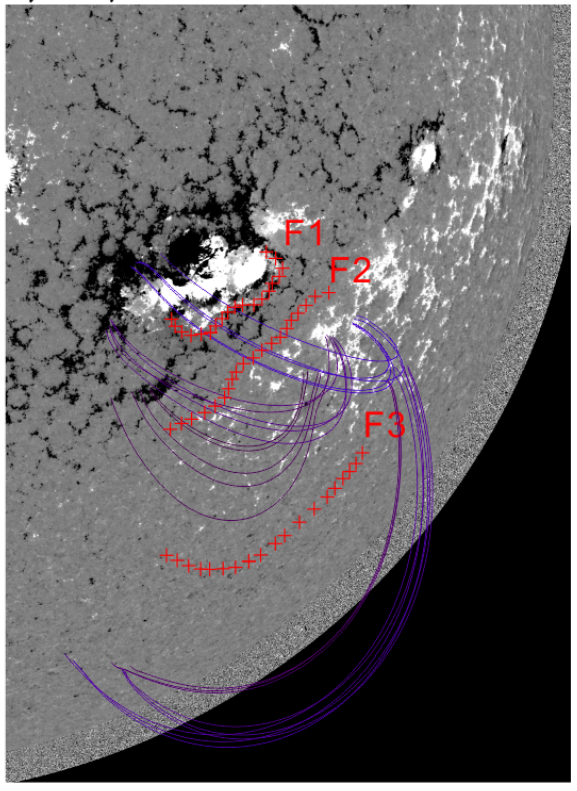

Figure 9. Schematic diagram of the slipping-like magnetic reconnection. a-c) The black thick lines indicate the filaments F2 and F3. The plus and minus signs denote positive and negative polarity, respectively. Magnetic field lines are denoted by thin lines, with black and red colors indicating those before and after reconnection, respectively. The red arrows mark the slipping directions observed on the surface as field lines are 'lightened' successively by magnetic reconnection. The red dots mark the footpoints of newly reconnected, closed field lines, indicating the brightening in the chromosphere and transition region. d) Representative PFSS field lines traced from the positive-polarity plage field between F2 and F3. The field lines are color-coded in the same way as in Figure 3. 